\begin{tabular}{|c|c|}
\hline Citation & $\begin{array}{l}\text { Dora Turk, Joris Gillis, Goele Pipeleers, Jan Swevers (2016), } \\
\text { Experimental Validation of a Combined Global and Local LPV System } \\
\text { Identification Approach with I2,1-norm Regularization } \\
\text { IECON } 2016 \text { - 42nd Annual Conference of the IEEE Industrial Electronics } \\
\text { Society, Florence, Italy, } 23 \text { - } 26 \text { October } 2016 \text {. }\end{array}$ \\
\hline Archived version & $\begin{array}{l}\text { Author manuscript: the content is identical to the content of the published } \\
\text { paper, but without the final typesetting by the publisher }\end{array}$ \\
\hline Published version & \\
\hline Journal homepage & http://ieeexplore.ieee.org/xpl/conhome.jsp?punumber $=1000352$ \\
\hline Author contact & $\begin{array}{l}\frac{\text { dora.turk@kuleuven.be }}{+32(0) 16372857}\end{array}$ \\
\hline IR & https://lirias.kuleuven.be/handle/123456789/555256 \\
\hline
\end{tabular}

(article begins on next page) 


\title{
Experimental Validation of a Combined Global and Local LPV System Identification Approach with $\ell_{2,1}$-norm Regularization*
}

\author{
Dora Turk ${ }^{1}$, Joris Gillis, Goele Pipeleers and Jan Swevers \\ Production Engineering, Machine Design and Automation, Department of Mechanical Engineering \\ KU Leuven, Belgium
}

\begin{abstract}
This paper explores a combined global and local identification approach for linear parameter-varying systems. Ideally, the combined approach retains advantages of its two extremes - global and local - with the possibility to emphasize one or the other. Practically, it is prone to overfitting. This paper proposes a remedy based on the $\ell_{2,1}$-norm regularization, describes its implementation within the nonlinear least squares framework, and gives an experimental validation. The results show a substantial decrease in the Euclidean norm of the model parameters, which resulted in a significantly smoother frequency response function surface and in overall, less-deviating model behavior.
\end{abstract}

Keywords - system identification, time-varying systems, mechatronics

\section{INTRODUCTION}

Linear parameter-varying (LPV) systems are nonlinear systems described by a linear model with coefficients varying as a function of one or more scheduling parameters. These time-varying parameters determine the system's operating point. The inherited features of the well-studied linear timeinvariant (LTI) systems make LPV systems attractive for modern industrial control, with main applications in aircrafts, robotics, and wind turbines.

The literature on LPV system identification distinguishes between a global and local approach. The global techniques (e.g. [1], [2]) directly identify an LPV model based on data obtained from an experiment where both the input signal and scheduling parameters are continuously changing. Experiments of this kind are referred to as global experiments. The local identification techniques (e.g. [3], [4]) typically consist of two steps. In the first step, several LTI models are identified based on local input-output data obtained for various fixed values of the scheduling parameters (local experiments). These LTI models are in the second step interpolated, yielding a parameter dependent model.

Both approaches have their advantages and disadvantages. The global approach aims at optimizing model accuracy under changing scheduling parameter conditions. In addition, dynamic scheduling dependency - dependency on timeshifted instances of the scheduling parameters - can only be

\footnotetext{
*This research is sponsored by the Fund for Scientific Research (FWO-Vlaanderen) through project G.0002.11, by the KU Leuven-BOF PFV/10/002 Center-of-Excellence Optimization in Engineering (OPTEC), and by the Belgian Program on Interuniversity Poles of Attraction, initiated by the Belgian State, Prime Ministers Office, Science Policy programming (IAP VII, DYSCO).

${ }^{1}$ dora.turk@kuleuven. be
}

detected through a global identification experiment. The local approach can only identify systems with static scheduling dependency - in which the system depends solely on the instantaneous time values of the scheduling parameters, but can to a large extent rely on the well-studied LTI identification methods. Although different, data originating from global and local experiments both provide valuable information that, when combined, gives a more complete picture of the system at hand. This hypothesis led to the combined global and local approach [5]. As a sequel, this paper validates the approach experimentally on a mechatronic XY-motion system. In addition, it provides an $\ell_{2,1}$-norm regularizationbased remedy for the problems observed in practice, and which can be attributed to an excess in degrees of freedom: a poor local model fit for the values of the scheduling parameter not used in the identification, a cumbersome model and frivolous evolution of the model's poles and zeroes.

The paper is organized as follows. Section II describes the chosen LPV model structure. Section III introduces the combined global and local identification method with $\ell_{2,1^{-}}$ norm regularization, shows the underlying optimization problem, and gives instructions for solving it. In Section IV, the presented identification method is experimentally validated and compared with the method without regularization [5]. The obtained results form the bottom line for the conclusions conveyed in Section V.

\section{LPV MODEL STRUCTURE}

In this paper we focus on the following fully parameterized discrete time LPV model:

$$
\left\{\begin{array}{l}
x(t+1)=(\mathscr{A} \diamond p)(t) \cdot x(t)+(\mathscr{B} \diamond p)(t) \cdot u(t) \\
y(t)=(\mathscr{C} \diamond p)(t) \cdot x(t)+(\mathscr{D} \diamond p)(t) \cdot u(t),
\end{array}\right.
$$

where $x(t) \in \mathbb{R}^{n}, u(t) \in \mathbb{R}^{r}, y(t) \in \mathbb{R}^{l}$, and $p(t) \in \mathbb{R}^{N_{p}}$, are respectively the state vector, the input vector, the output vector, and the scheduling parameter vector, at time instance $t$. The state-space matrices of the introduced model are parameter-dependent:

$$
\begin{aligned}
& (\mathscr{A} \diamond p)(t)=A_{0}+\sum_{i=1}^{N_{b}} A_{i} \psi_{i}\left(p(t), \ldots, p\left(t-n_{d}\right)\right), \\
& (\mathscr{B} \diamond p)(t)=B_{0}+\sum_{i=1}^{N_{b}} B_{i} \psi_{i}\left(p(t), \ldots, p\left(t-n_{d}\right)\right),
\end{aligned}
$$




$$
\begin{aligned}
& (\mathscr{C} \diamond p)(t)=C_{0}+\sum_{i=1}^{N_{b}} C_{i} \psi_{i}\left(p(t), \ldots, p\left(t-n_{d}\right)\right), \\
& (\mathscr{D} \diamond p)(t)=D_{0}+\sum_{i=1}^{N_{b}} D_{i} \psi_{i}\left(p(t), \ldots, p\left(t-n_{d}\right)\right),
\end{aligned}
$$

where $A_{0} \in \mathbb{R}^{n \times n}, A_{i} \in \mathbb{R}^{n \times n}, B_{0} \in \mathbb{R}^{n \times r}, B_{i} \in \mathbb{R}^{n \times r}, C_{0} \in$ $\mathbb{R}^{l \times n}, C_{i} \in \mathbb{R}^{l \times n}, D_{0} \in \mathbb{R}^{l \times r}, D_{i} \in \mathbb{R}^{l \times r} ; N_{b}$ is the number of basis functions $\psi_{i}$ employed for parameterization, and $n_{d}$ is the number of time-shifts of the scheduling parameters. It is here for brevity taken that $N_{b}$ and $n_{d}$ are equal for all model matrices $\{\mathscr{A}, \mathscr{B}, \mathscr{C}, \mathscr{D}\}$. This does not, however, have to always be the case.

\section{COMBINED GLOBAL AND LOCAL IDENTIFICATION APPROACH}

\section{A. Nonlinear least-squares problem}

Local identification data can be either time or frequency domain data. Global identification data are in most cases time domain data, although there are global identification methods that consider frequency domain data, e.g. [6], provided that the input and scheduling are chosen to be periodic and synchronized. In this paper, we only consider global identification data in time domain.

Assume that $N_{\mathrm{t}}$ different sets of time domain data and $N_{\mathrm{f}}$ different sets of frequency domain data are available.

First consider time domain data, which can originate from either local or global experiments. The difference between the response $\mathbf{y}^{q_{t}}(\Theta)$ of the LPV model (1) to the input of the $q_{\mathrm{t}}^{\text {th }}$ experiment, and the measured output $\mathbf{y}_{\mathrm{m}}^{q_{\mathrm{t}}}$, equals:

$$
\boldsymbol{\varepsilon}_{\mathrm{t}}^{q_{\mathrm{t}}}(\Theta)=\mathbf{y}^{q_{\mathrm{t}}}(\Theta)-\mathbf{y}_{\mathrm{m}}^{q_{\mathrm{t}}},
$$

where

$$
\Theta=[\operatorname{vec}(A) ; \operatorname{vec}(B) ; \operatorname{vec}(C) ; \operatorname{vec}(D)] .
$$

Second assume $N_{\mathrm{f}}$ local experiments providing frequency domain data. The difference between the system's complex freq response function (FRF) $\mathbf{G}_{\mathrm{m}}^{\mathbf{q}_{\mathrm{f}}}$ resulting from the $q_{\mathrm{f}}^{\text {th }}$ local experiment and the corresponding model FRF $\mathbf{G}^{q_{\mathrm{f}}}(\Theta)$ equals:

$$
\boldsymbol{\varepsilon}_{\mathrm{f}}^{q_{\mathrm{f}}}(\Theta)=\mathbf{G}^{q_{\mathrm{f}}}(\Theta)-\mathbf{G}_{\mathrm{m}}^{q_{\mathrm{f}}} .
$$

A (weighted) nonlinear least-squares (NLS) criterion that combines global and local experiments, from the time and frequency domain, can now be formulated:

$$
\begin{array}{r}
V_{\mathrm{NLS}}(\Theta)=\frac{1}{2}\left(\sum_{q_{\mathrm{t}}}\left(\boldsymbol{\varepsilon}_{\mathrm{t}}^{q_{\mathrm{t}}}(\Theta)\right)^{\mathrm{T}} W_{\mathrm{t}}^{q_{\mathrm{t}}} \boldsymbol{\varepsilon}_{\mathrm{t}}^{q_{\mathrm{t}}}(\Theta)+\right. \\
\left.\sum_{q_{\mathrm{f}}}\left(\boldsymbol{\varepsilon}_{\mathrm{f}}^{q_{\mathrm{f}}}(\Theta)\right)^{\mathrm{H}} W_{\mathrm{f}}^{q_{\mathrm{f}}} \boldsymbol{\varepsilon}_{\mathrm{f}}^{q_{\mathrm{f}}}(\Theta)\right) .
\end{array}
$$

The weighting matrices $W_{\mathrm{t}}$ and $W_{\mathrm{f}}$ serve to emphasize a time span or a frequency range of interest, respectively. In case no specific weighting is required, a constant that normalizes the time/frequency domain error is recommended, that is:

$$
W_{\mathrm{t}}^{q_{\mathrm{t}}}=\left(\sum_{q_{\mathrm{t}}}\left\|\mathbf{y}_{\mathrm{m}}^{q_{\mathrm{t}}}\right\|_{2}^{2}\right)^{-1}, W_{\mathrm{f}}^{q_{\mathrm{f}}}=\left(\sum_{q_{\mathrm{f}}}\left\|\mathbf{G}_{\mathrm{m}}^{q_{\mathrm{f}}}\right\|_{2}^{2}\right)^{-1} .
$$

The optimal set of parameter estimates $\Theta^{*}$ is then the one that minimizes (9), i.e.

$$
\Theta^{*}=\arg \min _{\Theta} V_{\mathrm{NLS}} .
$$

The solution of such a problem is typically obtained using the Levenberg-Marquardt algorithm.

\section{B. Second-Order Cone Programming (SOCP) problem}

Selecting an adequate set of basis functions is very challenging and time-consuming, particularly if no information on the scheduling parameter dependency of the system model is available. This issue has been widely discussed (see e.g. [7] and [8]). The approach chosen to tackle it in this paper is to propose a large set of basis functions based on physical insights and a trial-and-error procedure, and extract an adequate subset by applying $\ell_{2,1}$-norm regularization to the estimation problem. The $\ell_{2,1}$-norm of an arbitrary matrix $M \in \mathbb{R}^{m \times n}$ is defined as

$$
\|M\|_{2,1}=\sum_{j=1}^{n} \sqrt{\sum_{i=1}^{m} M(i, j)^{2}},
$$

and has a desirable "grouping" property: in case (12) would be added to an optimization problem where all elements of $M$ are optimization variables, the optimization favors solutions $M$ with as many zero columns as possible. In this paper we extend the concept of columns in (12) to the matrix blocks associated with the same basis functions, in order to obtain an algorithm that automatically discards redundant basis functions. The regularization term added to the NLS criterion (9) is therefore

$$
\begin{array}{r}
V_{\operatorname{reg}}(\Theta)=\gamma \sum_{i=1}^{N_{b}}\left(\left\|\operatorname{vec}\left(A_{i}\right)\right\|_{2}+\left\|\operatorname{vec}\left(B_{i}\right)\right\|_{2}+\right. \\
\left.+\left\|\operatorname{vec}\left(C_{i}\right)\right\|_{2}+\left\|\operatorname{vec}\left(D_{i}\right)\right\|_{2}\right),
\end{array}
$$

where $\gamma$ is a scalar, the value of which determines the importance of the regularization with regard to the model accuracy, and vec stands for matrix vectorization. The optimal set of parameters is now one that minimizes the updated criterion:

$$
\Theta^{*}=\arg \min _{\Theta}\left(V_{\mathrm{NLS}}(\Theta)+V_{\mathrm{reg}}(\Theta)\right) .
$$

Due to a nonquadratic nature of (13), the optimization problem (14) cannot be solved by the Levenberg-Marquardt algorithm. However, having it reformulated into

$$
\begin{array}{cl}
\underset{\Theta, s}{\operatorname{minimize}} & V_{\mathrm{NLS}}(\Theta)+\gamma \sum_{i=1}^{N_{b}}\left(s_{i}^{A}+s_{i}^{B}+s_{i}^{C}+s_{i}^{D}\right) \\
\text { subject to } & \left\|\operatorname{vec}\left(A_{i}\right)\right\|_{2} \leq s_{i}^{A} \\
& \left\|\operatorname{vec}\left(B_{i}\right)\right\|_{2} \leq s_{i}^{B} \\
& \left\|\operatorname{vec}\left(C_{i}\right)\right\|_{2} \leq s_{i}^{C} \\
& \left\|\operatorname{vec}\left(D_{i}\right)\right\|_{2} \leq s_{i}^{D} \\
& i=1, \ldots, N_{b}
\end{array}
$$


one can recognize a nonlinear second-order cone programming (NSOCP) problem, [9]. In [10], an SQP-type algorithm for solving such problems is proposed. This algorithm solves a convex SOCP subproblem in each iteration, with the constraints being linear approximations of the constraint functions of the original problem, and with a convex quadratic function as the objective function. The same principle is adopted here, but remaining withing the LevenbergMarquardt framework. Namely, in each iteration $k$ the step $\Delta \Theta$ is calculated by solving the following subproblem

$$
\begin{array}{rr}
\underset{\Delta \Theta, \Delta s}{\operatorname{minimize}} & \nabla V_{\mathrm{NLS}}\left(\Theta^{k}\right)^{T} \Delta \Theta+\frac{1}{2} \Delta \Theta^{T} M_{k} \Delta \Theta+ \\
+ & \gamma \sum_{i=1}^{N_{b}}\left(\Delta s_{i}^{A}+\Delta s_{i}^{B}+\Delta s_{i}^{C}+\Delta s_{i}^{D}\right) \\
\text { subject to } \quad & \left\|\operatorname{vec}\left(A_{i}^{k}+\Delta A_{i}^{k}\right)\right\|_{2} \leq s_{i}^{A, k}+\Delta s_{i}^{A} \\
& \left\|\operatorname{vec}\left(B_{i}^{k}+\Delta B_{i}^{k}\right)\right\|_{2} \leq s_{i}^{B, k}+\Delta s_{i}^{B} \\
& \left\|\operatorname{vec}\left(C_{i}^{k}+\Delta C_{i}^{k}\right)\right\|_{2} \leq s_{i}^{C, k}+\Delta s_{i}^{C} \\
& \left\|\operatorname{vec}\left(D_{i}^{k}+\Delta D_{i}^{k}\right)\right\|_{2} \leq s_{i}^{D, k}+\Delta s_{i}^{D} \\
i=1, \ldots, N_{b}
\end{array}
$$

using the Embedded Conic Solver (ECOS) [11]. In (16), $M_{k}$ is a Hessian approximation matrix defined as in the original Levenberg-Marquardt version:

$$
\begin{array}{r}
M_{k}=\nabla V_{\mathrm{NLS}}\left(\Theta^{k}\right)^{T} \nabla V_{\mathrm{NLS}}\left(\Theta^{k}\right)+ \\
+\lambda^{2} \operatorname{diag}\left(\nabla V_{\mathrm{NLS}}\left(\Theta^{k}\right)^{T} \nabla V_{\mathrm{NLS}}\left(\Theta^{k}\right)\right),
\end{array}
$$

where $\nabla V_{\mathrm{NLS}}\left(\Theta^{k}\right)$ is the Jacobian matrix, and $\lambda$ is the damping parameter. The algorithm is stopped once the step size is smaller than a specified threshold or after a sufficient improvement in the model performance has been reached.

\section{EXPERIMENTAL VALIDATION: IDENTIFICATION OF AN XY-MOTION SYSTEM}

\section{A. Setup description}

The system under test is the XY-motion system shown in Fig. 1. The system consists of two perpendicularly mounted linear stages (X and $\mathrm{Y}$ ) and a flexible cantilever beam. The length of this beam is changed by the position of the Ymotor, such that the cantilever beam resonances and hence the dynamics of the XY-motion system in the X-direction depends on the position of the Y-motor [12]. The reference position for the position controller of the Y-motor can thus be seen as a scheduling parameter of the system we aim to identify. The reference velocity for the velocity controller of the X-motor is the system input, while the acceleration of the end-effector in the same direction represents the system output. The acceleration is measured by a MEMS (Micro-Electro-Mechanical Systems) accelerometer designed to measure low frequency vibration and motion, and having a flat frequency spectrum within $f \in[0,250] \mathrm{Hz}$. The estimated signal-to-noise (SNR) ratio is $47 \mathrm{~dB}$.

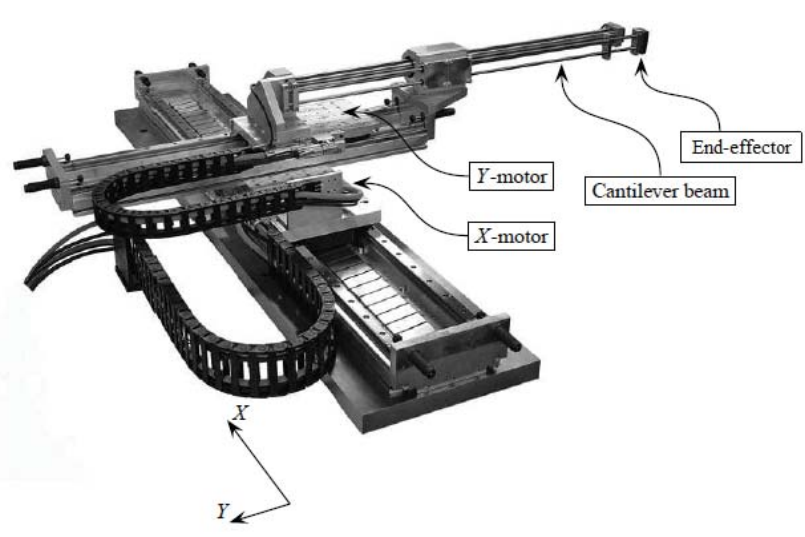

Fig. 1. XY-motion system

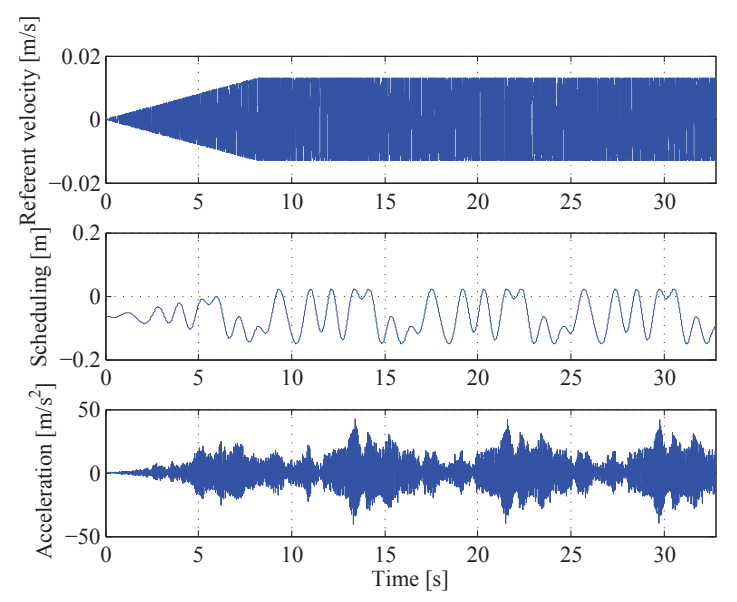

Fig. 2. Global data set used for identification.

\section{B. Experiment design}

The assessment of the proposed approach involves global and local experiments. All experiments were executed at sampling rate $f_{s}=1 \mathrm{kHz}$. In the global experiment, the system input was excited with a random-phase multisine signal, [13], composed of frequencies in the range $f \in[3,50] \mathrm{Hz}$. The amplitude of this signal was selected to avoid motor current saturation. The scheduling signal is a random multisine as well, with the same period as the system input, that is 8.192 seconds, however with a much more restricted frequency spectrum $f \in[0.1,1] \mathrm{Hz}$ and taking values in the operating range $p \in[-0.1508,0.0229] \mathrm{m}$, where $0 \mathrm{~m}$ corresponds to the middle position of the stage. A global data set then consists of four consecutive signal periods, each comprising 8192 data samples of the reference velocity for the X-motor, measured position of the Y-motor and the resulting acceleration of the end-effector. The first period differs from the others in the sense that the input is scaled by a ramp signal for a smooth start. Two different realizations of the global experiment were conducted, one for identification (Fig. 2) and the other for validation. The local experiments were performed for fixed values of the scheduling parameter, that is, fixed positions of the Y-motor. Four local experiments, 
for the positions of the Y-motor equal to

$$
p=-0.1508,-0.0929,-0.0350,0.0229 \mathrm{~m}
$$

were performed. The X-motor input signal for each local experiment is a random multisine with the same specifications as for the global experiments. The local identification data are chosen to be used in the frequency domain. The local data set consists of four frequency response functions (FRFs), corresponding to four experiments and evaluated at 385 equally distributed frequency lines of interest $(f \in[3,50] \mathrm{Hz})$.

\section{Algorithm settings}

The presented identification method requires an initial estimate of the LPV model parameters (7). This is provided by the SMILE technique (State-space Model Interpolation of Local Estimates) presented in [12], a numerically wellconditioned local identification technique based on the interpolation of a set of local LTI models that are obtained for fixed operating conditions of the system. In our case, these LTI models are obtained using a nonlinear least-squares frequency domain linear model identification method [13]. The LTI models are of the fourth order, which dictates the order of the LPV model. Since there was no apriori knowledge about suitable basis functions, and since there are four LTI models to be interpolated, a third-order polynomial scheduling parameter dependency and hence following set of basis functions:

$$
\psi_{1}=p(t), \quad \psi_{2}=p(t)^{2}, \quad \psi_{3}=p(t)^{3},
$$

was chosen. Through this choice, the interpolation can be performed without introducing errors, that is, the LTI models correspond exactly to the LPV model for the corresponding fixed values of the scheduling parameter. Global and local identification data are combined into the objective function (9), with $W_{\mathrm{t}}$ and $W_{\mathrm{f}}$ as in (10). The global and local data are given the same importance. Both the NLS combined global and local approach [5] minimizing (9), and its regularized version $\mathrm{NLS}_{\ell_{2,1}}$ solving (15) with $\gamma=0.1$, are applied.

\section{Results}

In the first case, the Levenberg-Marquardt algorithm did not converge; it was stopped because the maximum number of iterations (1000) had been reached. The sequential SOCP was stopped after 50 iterations. By looking at Fig. 3 and Table I, one can see a significant improvement in the global model accuracy achieved with the NLS model (yellow), and with the $\mathrm{NLS}_{\ell_{2,1}}$ (green) model, in comparison with the SMILE model both algorithms start from (red). This is expected given the local nature of the SMILE technique. It also justifies the use of the global data in addition to the local. When compared to the NLS model, the $\mathrm{NLS}_{\ell_{2,1}}$ model gave $(\approx 35 \%)$ larger global error; such an outcome, although unwelcome, should not surprise since there was also the regularization term taken into account.

Fig. 4 and Fig. 5 evaluate the models using the local identification data (FRF measurements). The SMILE model performs better than the NLS and $\mathrm{NLS}_{\ell_{2,1}}$ models, expectedly
TABLE I

ROOT MEAN SQUARE ERROR $\left[\mathrm{m} / \mathrm{s}^{2}\right.$ ] OF THE MODELS ON GLOBAL IDENTIFICATION AND VALIDATION DATA

\begin{tabular}{cccc} 
Data set & SMILE & NLS & NLS $_{\ell_{2,1}}$ \\
\hline Identification & 5.6939 & 1.7859 & 2.4738 \\
\hline Validation & 3.7841 & 1.7439 & 2.2969 \\
\hline
\end{tabular}

since it originates from a local technique. The NLS model is slightly more accurate than the $\mathrm{NLS}_{\ell_{2,1}}$ model. However, Fig. 6 and Fig. 7, which portray the dependence of the model FRF on the scheduling parameter, show unnatural behaviour of the NLS model for the scheduling parameter values not involved in the identification (note that the FRF surface of the SMILE model was smooth). There are undesired sudden variations of the magnitude and phase surface, resulting in a poor overall fit in the local sense. The $\mathrm{NLS}_{\ell_{2,1}}$ does not have that problem, which can be seen in Fig. 8 and Fig. 9.

Fig. 10 depicts the frivolous pole-zero evolution of the

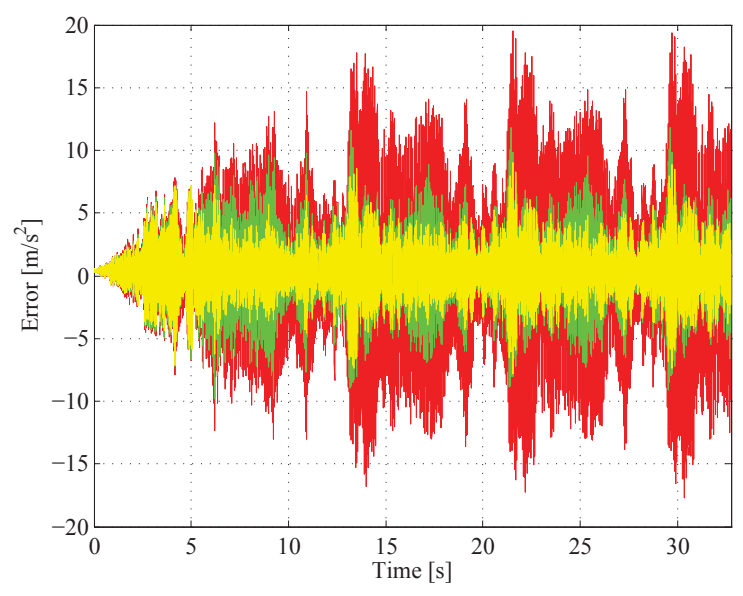

Fig. 3. Global identification error of the SMILE model (red), the NLS model (yellow), and the $\mathrm{NLS}_{\ell_{2,1}}$ model (green).

NLS model as the scheduling parameter is changing from the minimal to the maximal operating value. The sudden changes in values of the poles and zeroes are a possible explanation for the bumpy magnitude and phase surfaces. What goes in favor of such reasoning are the neatly grouped poles and zeroes of the $\mathrm{NLS}_{\ell_{2,1}}$ model (Fig. 11).

Table II and III give an indication of the size of the parameters forming the state-space matrices of the NLS and $\mathrm{NLS}_{\ell_{2,1}}$ model, respectively. Table II shows that the values of the parameters in the NLS model are scattered throughout a large range, and throughout the whole model, making it not that well conditioned. Table III unveils that the size of parameters in $B_{i}, C_{i}, D_{i},(i=1,2,3)$ is significantly smaller than the size of the parameters in $A_{0}, B_{0}, C_{0}, D_{0}$, meaning that the belonging basis functions are there unnecessary. The $\ell_{2,1}$ regularization thus enabled us to select a simpler scheduling parameter dependency, in the sense that the input matrix $(\mathscr{B})$ and the complete output equation (matrices $\mathscr{C}$ and $\mathscr{D}$ ) can be modeled as being scheduling parameter independent. 


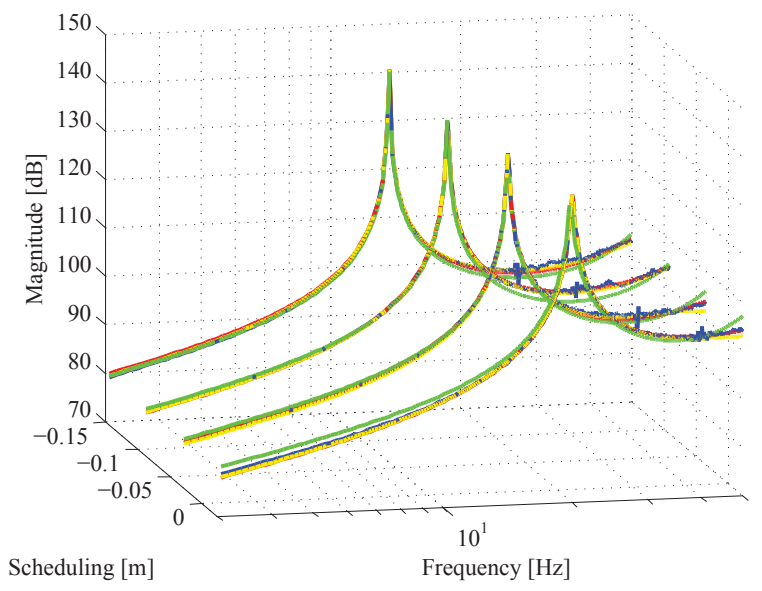

Fig. 4. Local identification fit - magnitude. Blue - the measurements, red the SMILE model, yellow - the NLS model, and green - the $\mathrm{NLS}_{\ell_{2,1}}$ model.

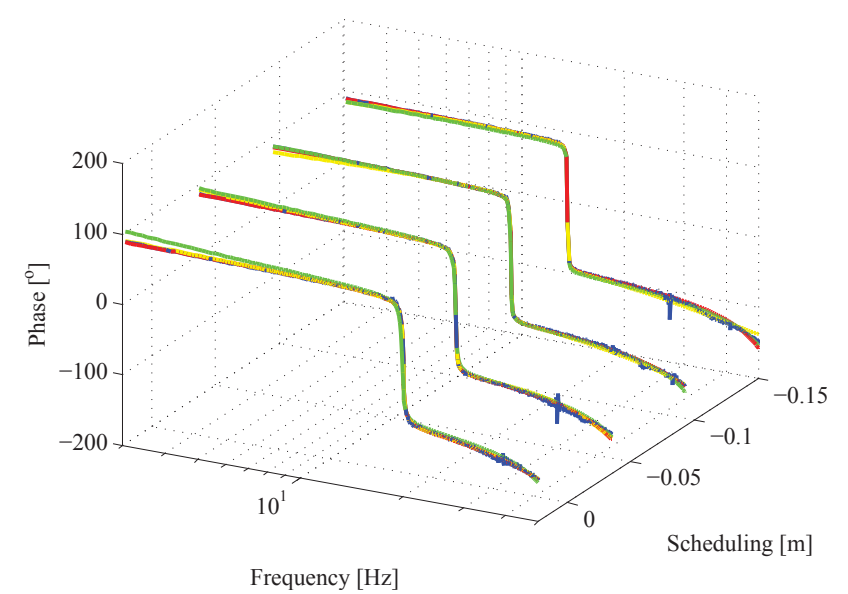

Fig. 5. Local identification fit - phase. Blue - the measurements, red - the SMILE model, yellow - the NLS model, and green - the $\mathrm{NLS}_{\ell_{2,1}}$ model.

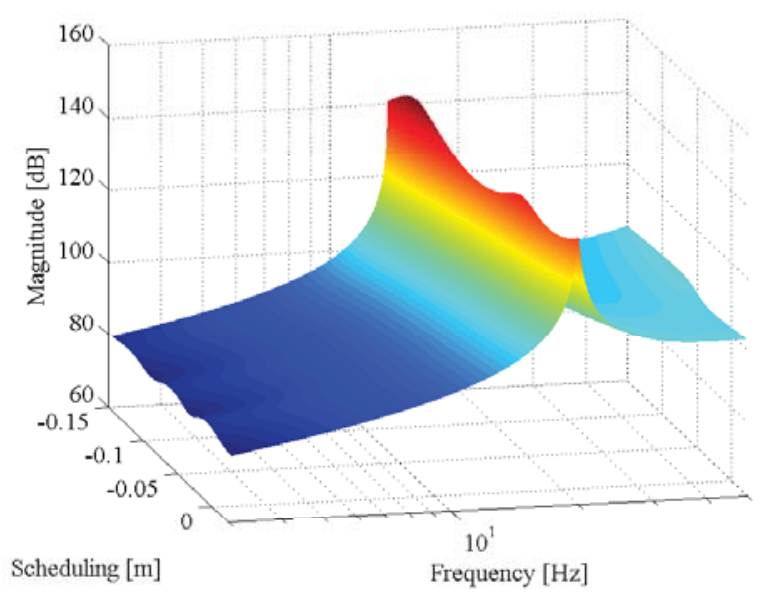

Fig. 6. Magnitude surface of the NLS model.

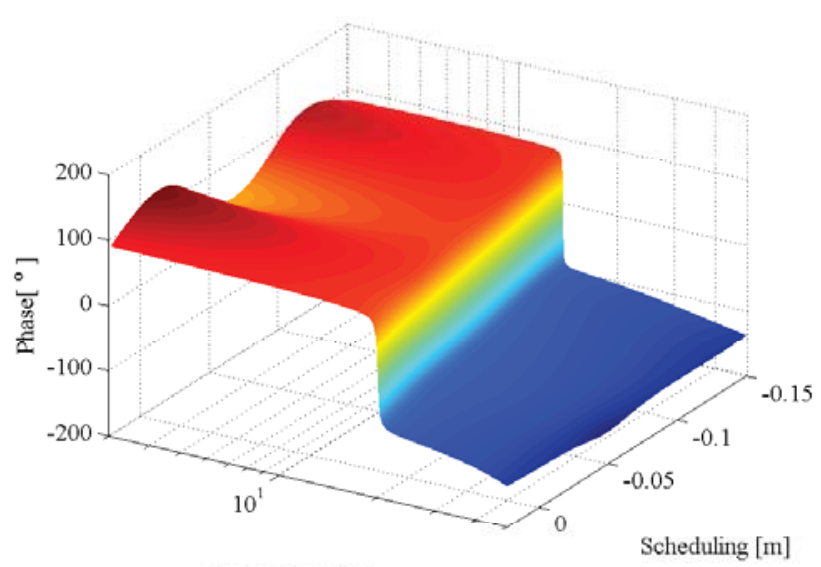

Frequency $[\mathrm{Hz}]$

Fig. 7. Phase surface of the NLS model.

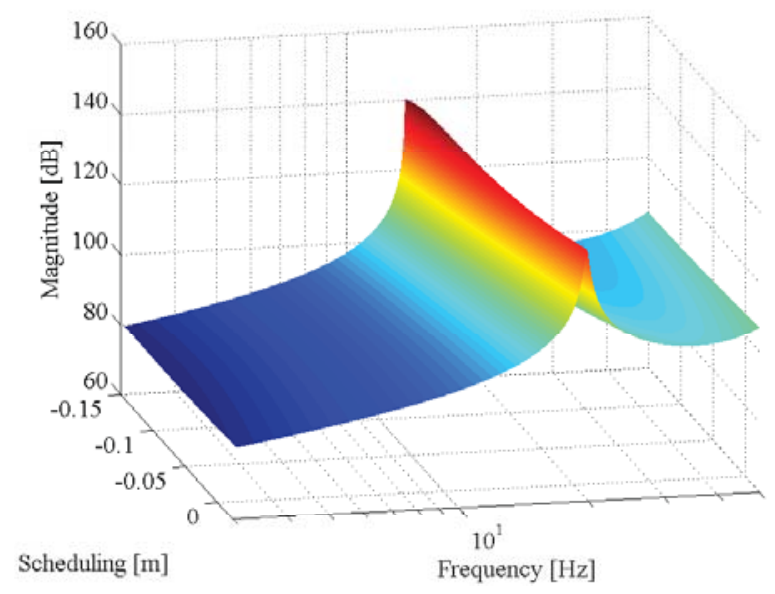

Fig. 8. Magnitude surface of the $\mathrm{NLS}_{\ell_{2,1}}$ model.

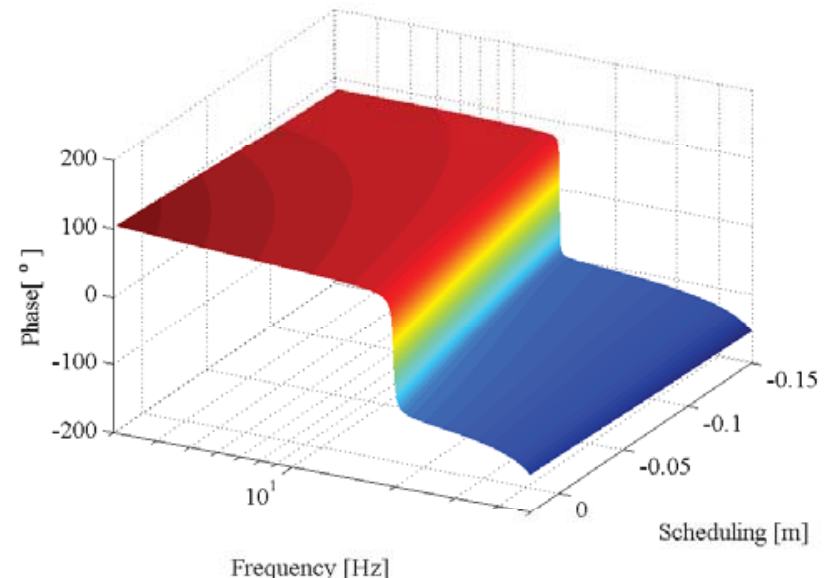

Fig. 9. Phase surface of the $\mathrm{NLS}_{\ell_{2,1}}$ model. 
TABLE II

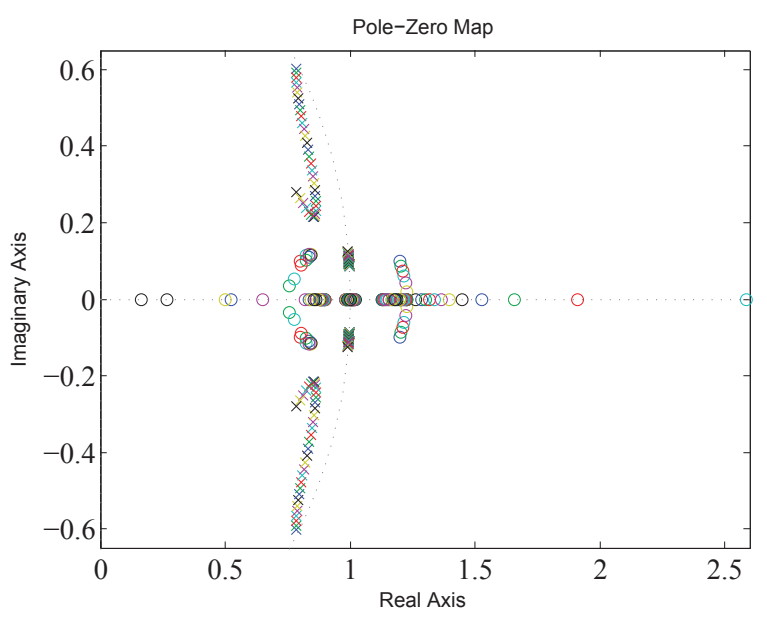

Fig. 10. Pole (x) - zero (o) evolution of the NLS model, over the operating range.

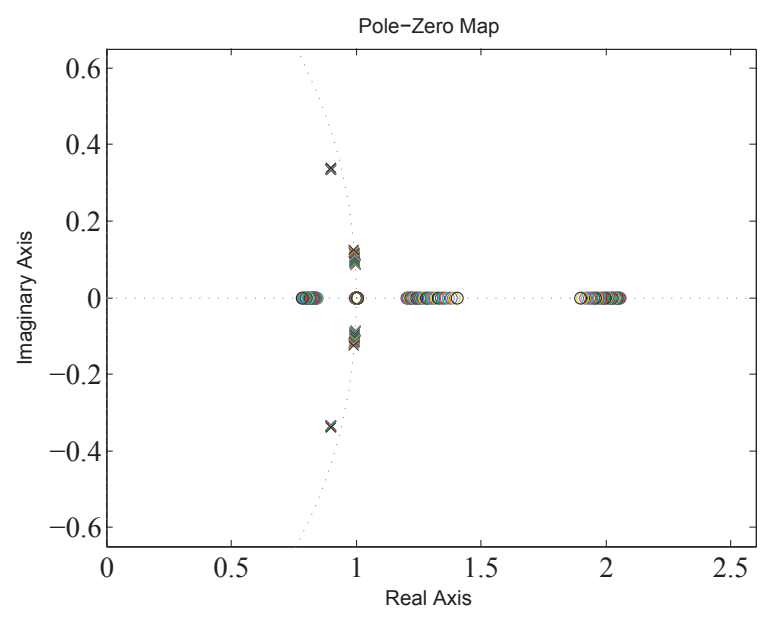

Fig. 11. Pole (x) - zero (o) evolution of the model obtained with the $\mathrm{NLS}_{\ell_{2,1}}$ model, over the operating range.

Keeping in mind the importance of well-conditioning and sparsity in the LPV control design, the proposed regularized NLS-based LPV identification method shows a potential.

\section{CONCLUSION}

This paper gives practical insights into identification of LPV systems via the combined global and local approach by revealing potential difficulties and proposing a way to deal with them. A substantial number of model parameters and accordingly degrees of freedom - stimulates occasional wanderings of the Levenberg-Marquardt algorithm, resulting in cumbersome models. A consequence we experienced when identifying a mechatronic system was unexpected model behavior for the scheduling values that are in-between the ones used in the identification, manifested in a nonsmooth FRF surface and pole-zero evolution. To eliminate this effect, we explored the $\ell_{2,1}$-norm regularization of the model parameters aiming at reducing possible overfitting.
PARAMETER SIZE OF THE NLS MODEL

\begin{tabular}{ccccc}
$i$ & $\left\|\operatorname{vec}\left(A_{i}\right)\right\|_{2}$ & $\left\|\operatorname{vec}\left(B_{i}\right)\right\|_{2}$ & $\left\|\operatorname{vec}\left(C_{i}\right)\right\|_{2}$ & $\left\|\operatorname{vec}\left(D_{i}\right)\right\|_{2}$ \\
\hline 0 & 2.6238 & 0.7556 & $1.2796 \cdot 10^{5}$ & $6.9043 \cdot 10^{4}$ \\
1 & 4.7264 & 0.0189 & $9.8387 \cdot 10^{5}$ & $3.8269 \cdot 10^{5}$ \\
2 & 78.3631 & 0.0574 & $1.2211 \cdot 10^{7}$ & $3.3724 \cdot 10^{7}$ \\
3 & 331.0831 & 2.6293 & $4.4526 \cdot 10^{7}$ & $1.1201 \cdot 10^{8}$ \\
\hline
\end{tabular}

TABLE III

PARAMETER SIZE OF THE $\mathrm{NLS}_{\ell_{2,1}}$ MODEL

\begin{tabular}{ccccc}
$i$ & $\left\|\operatorname{vec}\left(A_{i}\right)\right\|_{2}$ & $\left\|\operatorname{vec}\left(B_{i}\right)\right\|_{2}$ & $\left\|\operatorname{vec}\left(C_{i}\right)\right\|_{2}$ & $\left\|\operatorname{vec}\left(D_{i}\right)\right\|_{2}$ \\
\hline 0 & 2.7656 & 9.7760 & $9.1592 \cdot 10^{4}$ & $1.2223 \cdot 10^{4}$ \\
1 & 0.0845 & $1.5277 \cdot 10^{-10}$ & $6.6762 \cdot 10^{-12}$ & $2.2975 \cdot 10^{-12}$ \\
2 & 0.1321 & $1.2057 \cdot 10^{-10}$ & $2.8741 \cdot 10^{-11}$ & $2.2942 \cdot 10^{-12}$ \\
3 & 0.0194 & $1.0018 \cdot 10^{-10}$ & $3.7488 \cdot 10^{-11}$ & $2.3856 \cdot 10^{-12}$ \\
\hline
\end{tabular}

Reformulation of the optimization problem into a NSOCP that is sequentially solved in each Levenberg-Marquardt iteration, resulted in an approach we here proposed and which showed successful in identifying the XY-motion system. Future extensions will focus on regaining the accuracy of the nonregularized solution, while keeping the model sparse.

\section{REFERENCES}

[1] B. Bamieh and L. Giarre, "Identification of linear parameter varying models," International Journal of Robust and Nonlinear Control, vol. 12 , no. 9, pp. 841-853, 2002.

[2] F. Felici, J.-W. Van Wingerden, and M. Verhaegen, "Subspace identification of MIMO LPV systems using a periodic scheduling sequence," Automatica, vol. 43, no. 10, pp. 1684-1697, 2007.

[3] J. De Caigny, R. Pintelon, J. Camino, and J. Swevers, "Interpolated modeling of LPV systems based on observability and controllability," in Proc. of the 16th IFAC Symposium on System Identification, Brussels, Belgium, pp. 1773-1778, 2013.

[4] M. Steinbuch, R. Van De Molengraft, and A. van der Voort, "Experimental modelling and LPV control of a motion system," in Proceedings of the American Control Conference, 2003, vol. 2, pp. 1374-1379, IEEE, 2003.

[5] D. Turk, G. Pipeleers, and J. Swevers, "A combined global and local identification approach for LPV systems," IFAC-PapersOnLine, vol. 48, no. 28, pp. 184-189, 2015.

[6] J. Goos, J. Lataire, and R. Pintelon, "Estimation of linear parametervarying affine state space models using synchronized periodic input and scheduling signals," in American Control Conference (ACC), 2014, pp. 3754-3759, IEEE, 2014.

[7] R. Tóth, H. Hjalmarsson, and C. R. Rojas, "Order and structural dependence selection of LPV-ARX models revisited," in Decision and Control (CDC), 2012 IEEE 51st Annual Conference on, pp. 62716276, IEEE, 2012.

[8] P. Gebraad, J.-W. van Wingerden, and M. Verhaegen, "Sparse estimation for predictor-based subspace identification of LPV systems," in 16th IFAC Symposium on System Identification, pp. 1749-1754, 2012.

[9] M. S. Lobo, L. Vandenberghe, S. Boyd, and H. Lebret, "Applications of second-order cone programming," Linear algebra and its applications, vol. 284, no. 1, pp. 193-228, 1998.

[10] H. Kato and M. Fukushima, "An SQP-type algorithm for nonlinear second-order cone programs," Optimization Letters, vol. 1, no. 2, pp. 129-144, 2007.

[11] A. Domahidi, E. Chu, and S. Boyd, "ECOS: An SOCP solver for embedded systems," in European Control Conference (ECC), pp. 30713076, 2013.

[12] J. De Caigny, J. F. Camino, and J. Swevers, "Interpolating model identification for SISO linear parameter-varying systems," Mechanical Systems and Signal Processing, vol. 23, no. 8, pp. 2395-2417, 2009.

[13] R. Pintelon and J. Schoukens, System identification: a frequency domain approach. John Wiley \& Sons, 2012. 